Methods A secondary cross-sectional data analysis was performed using National Survey on Drug Use and Health (NSDUH) data from 2015 to 2017 and including 126,463 individuals with 8,241 identifying as SM. The NSDUH is an annual, nationally representative survey of the civilian, noninstitutionalised US population aged twelve or older which estimates the prevalence and associated determinants of substance use and mental illness. Multivariable logistic regression models were used to quantify disparities in substance use among SM and to examine its link with sociodemographic factors and mental health.

Results SM showed higher levels of past-year substance use, misuse of prescription drugs and lifetime chemsex drug use relative to heterosexuals. Gay men showed the highest level of chemsex drug use. Bisexual women exhibited higher levels of use of cocaine, crack, heroin, methamphetamine and misuse of benzodiazepines relative to heterosexual women. Among women, lesbians showed higher odds for use of chemsex drugs and misuse of Oxycontin. Urbanity, being uninsured, and having an unmet need for mental health treatment were associated with significantly higher levels of substance use, including chemsex drug use. SM also experienced significantly higher levels of socioeconomic vulnerability, which were associated with increased odds for drug use.

Conclusion This study is among the first nationally representative samples that analysed the link between sociodemographic factors and unmet need for mental health treatment on substance use among SM. It emphasises the multifactorial aetiology of vulnerability to substance use and highlights the distinct disparities in, and underlying mechanisms for substance use among SM. Higher levels of substance use and misuse observed among SM have public health implications due to the negative health outcomes they may involve in the longterm. Of particular interest is the link established between substance use and mental health issues, considering the lack of tailored approaches aimed at addressing comorbidities and specific health needs of SM, especially given the structural barriers (i.e., stigma, discrimination and criminalisation of substance use) those populations face in accessing health services. Critical gaps in the literature remain and large-scale studies inclusive of SM individuals are needed to establish causal links.

\section{P71 LONELINESS AND PERSONAL WELLBEING IN YOUNG PEOPLE: THE MODERATING EFFECTS OF SOCIAL WELLBEING AND SOCIO-DEMOGRAPHIC FACTORS}

Emily Long*, Claire Goodfellow. MRC/CSO Social and Public Health Sciences Unit, University of Glasgow, Glasgow, UK

\subsection{6/jech-2021-SSMabstracts.159}

Background Loneliness a growing public health concern. This is particularly so in light of the Covid-19 pandemic which has highlighted the detrimental psychosocial consequences of loneliness. Loneliness is a particularly pervasive problem among young adults, but despite this, most research examining loneliness is among older adults. Loneliness is a known risk factor for poor health and personal wellbeing. However, the extent to which other aspects of social wellbeing (e.g., isolation, social support) may mitigate the relationship between loneliness and personal wellbeing is unclear. Loneliness is often used interchangeably with related, yet distinct aspects of social wellbeing, such as isolation. Therefore, it is difficult to differentiate factors that relate to loneliness, factors that relate to other components of social wellbeing, and the possible interactions between these constructs. Consequently, we aim to examine the interplay of loneliness and isolation on personal wellbeing.

Methods We make use of cross-sectional sample of 965 young people aged 16-24 from the 2018 wave of the Community Life Survey to conduct regression-based analyses. This allows us to evaluate for a direct effect of loneliness on personal wellbeing, and for an interaction effect between loneliness and isolation to determine if the presence of both loneliness and isolation is predictive of poorer wellbeing. Finally, we use moderated regression to assess whether individual, social, and community level factors influence the relationship between loneliness and personal wellbeing.

Results Preliminary results identify that loneliness is consistently associated with poorer personal wellbeing among young people. Isolation neither predicts wellbeing, nor moderates associations between loneliness and wellbeing. Factors such as trust in one's neighbourhood, not acting as a carer, and being a fulltime student were associated with greater wellbeing. At the individual level, a moderating effect of sex was found, and social factors (e.g., being able to count on friends) moderated the association between loneliness and wellbeing.

Conclusion Results suggests that the presence of both loneliness and isolation does not increase risk of poor personal wellbeing among young people. Rather, the subjective experience of loneliness is independently detrimental to wellbeing. Our results also identified that being of female gender was associated with increased risk of loneliness impacting on personal wellbeing, but that strong emotional support may act as a protective factor against loneliness, and therefore improve personal wellbeing. It is also important to foster community trust and engagement to improve wellbeing, and that young people with caring responsibilities may be particularly at risk of low personal wellbeing.

\section{P72 \\ ONLINE GAMING AND WELL-BEING IN THE ENGLISH LONGITUDINAL STUDY OF AGEING}

${ }^{1}$ Pamela Almeida-Meza*, ${ }^{1}$ Dorina Cadar, ${ }^{1}$ Andrew Steptoe, ${ }^{2}$ Carrie Ryan. ${ }^{1}$ Department of Behavioural Science and Health, University College London, London, UK; ${ }^{2}$ Department of Anthropology, University College London, London, UK

\subsection{6/jech-2021-SSMabstracts. 160}

Background Play and games are considered important contributors to healthy ageing; however, it is not clear how online gaming contributes to this association. We aimed to explore the impact of online gaming on older adult well-being.

Methods Using data from 3,067 participants aged 50 years and older from the English Longitudinal Study of Ageing, we performed multiple regressions and logistic regressions to assess the association between online gaming at (wave 6, 2012/13) and quality-of-life, loneliness, and depression at (wave 9, 2018/19) over a follow up of up to 7 years. Quality-of-life was assessed using the CASP-19 scale, loneliness was assessed using the UCLA Loneliness scale, and depression was assessed using the Centre for Epidemiologic Studies Depression Scale. Adjustment for covariates was carried out gradually: Model 1 adjusted for age, sex and marital status, model 